\title{
Comparison of Definition of Several Fractional Derivatives
}

\author{
Yun Ouyang ${ }^{1, a}$ and Wusheng Wang ${ }^{1, b^{*}}$ \\ ${ }^{1}$ School of Mathematics and Statistics, Hechi University, Guangxi, Yizhou 546300, P. R. China \\ a shuxueoyy@126.com, bwang4896@126.com \\ ${ }^{*}$ The corresponding author
}

Keywords: Riemann-liouville fractional derivatives; Caputo's fractional derivative; Grunwald-letnikov fractional derivatives; Fractional integral.

\begin{abstract}
The idea of fractional derivatives was raised first by L'Hospital in 1695. The fractional calculus and its mathematical consequences attracted many mathematicians such as Fourier, Euler, Laplace. Various definitions of non-integer order integral or derivative was given by many mathematicians. In this paper, firstly, we discuss the positive integer higher order derivative of a function, and obtain general formula of high order derivative. Secondly, we introduce the definitions of Grl"unwald-Letnikov, Riemann-Liouville and Caputo. Finally, we point out the relationship between these definitions. Caputo's integral definition and Grl"unwald-Letnikov integral definition are consistent with the Riemann-Liouville integral definition. When $f$ has $m+1$ order continuous derivative and $m<p<m+1$, Grl"unwald-Letnikov positive non-integer order derivative definition is consistent with the Riemann-Liouville positive non-integer order derivative definition.
\end{abstract}

\section{Introduction}

The idea of fractional derivatives was raised first by L'Hospital in 1695. The fractional calculus and its mathematical consequences attracted many mathematicians such as Fourier, Euler, Laplace. Various definitions of non-integer order integral or derivative was given by many mathematicians (see [1]). For fractional derivative, the most popular definitions are Riemann- Liouville and Caputo definitions as follows, respectively. Recently, the fractional calculus methods have become increasingly more popular and successful in a large number of physical applications (see for example, [2, 3]). The theoretical and experimental scientists observed that certain materials admit anomalous, fractional behavior, as [4]. There are many systems which evolution is governed by some fractional differential equations (for example, [5]). Fractional calculus is nowadays a broad and interesting field of very active research (see [6-12]).

In this paper, firstly, we discuss the positive integer high order derivative of a function, and obtain general formula of high order derivative. Secondly, we introduce the definitions of Grl" unwald-Letnikov, Riemann-Liouville and Caputo. Finally, we point out the relationship between these definitions.

\section{General Formula of High Order Derivative}

Suppose that function $f(t)$ has $n$ order continuous differentiable derivative on $[a, b]$. From the definition of the derivative, we have

$$
\begin{aligned}
& f^{\prime}(t)=\frac{d f}{d t}=\lim _{h \rightarrow 0} \frac{f(t)-f(t-h)}{h}, \\
& f^{\prime \prime}(t)=\frac{d^{2} f}{d t^{2}}=\lim _{h \rightarrow 0} \frac{f^{\prime}(t)-f^{\prime}(t-h)}{h}
\end{aligned}
$$




$$
\begin{aligned}
& =\lim _{h \rightarrow 0} \frac{1}{h}\left\{\frac{f(t)-f(t-h)}{h}-\frac{f(t-h)-f(t-2 h)}{h}\right\} \\
& =\lim _{h \rightarrow 0} \frac{f(t)-2 f(t-h)+f(t-2 h)}{h^{2}},
\end{aligned}
$$

By induction we can obtain

$$
f^{(n)}(t)=\frac{d^{n} f}{d t^{n}}=\lim _{h \rightarrow 0} \frac{1}{h^{n}} \sum_{r=0}^{n}(-1)^{r}\left(\begin{array}{l}
n \\
r
\end{array}\right) f(t-r h),
$$

where $n$ is a natural number,

$$
\left(\begin{array}{l}
n \\
r
\end{array}\right)=\frac{n(n-1)(n-2) \cdots(n-r+1)}{r !}
$$

Let $p$ be any arbitrary integer, define a new derivative (see $[9,10])$

$$
{ }_{a} D_{t}^{-p} f(t)=\lim _{\substack{h \rightarrow 0 \\
n h=t-a}} h^{p} \sum_{r=0}^{n}(-1)^{r}\left(\begin{array}{l}
p \\
r
\end{array}\right) f(t-r h),
$$

where $h=(t-a) / n$,

$$
\left(\begin{array}{l}
p \\
r
\end{array}\right)=\frac{p(p-1)(p-2) \cdots(p-r+1)}{r !} .
$$

If $p<0$, let $q=-p$,

$$
\left[\begin{array}{l}
q \\
r
\end{array}\right]=\frac{q(q+1)(q+2) \cdots(q+r-1)}{r !},
$$

then

$$
\left(\begin{array}{l}
p \\
r
\end{array}\right)=\frac{-q(-q-1)(-q-2) \cdots(-q-r+1)}{r !}=(-1)^{r}\left[\begin{array}{l}
q \\
r
\end{array}\right],
$$

or,

$$
(-1)^{r}\left(\begin{array}{l}
p \\
r
\end{array}\right)=\left[\begin{array}{l}
q \\
r
\end{array}\right] .
$$

From (5) and (9), we have

$$
{ }_{a} D_{t}^{q} f(t)=\lim _{\substack{h \rightarrow 0 \\
n h=t-a}} h^{q} \sum_{r=0}^{n}\left[\begin{array}{l}
q \\
r
\end{array}\right] f(t-r h), \quad p<0, q=-p>0 .
$$

In particular, we have

$$
\begin{aligned}
{ }_{a} D_{t}^{-1} f(t) & =\lim _{\substack{h \rightarrow 0 \\
h h=t-a}} h \sum_{r=0}^{n}\left[\begin{array}{l}
1 \\
r
\end{array}\right] f(t-r h) \\
& =\lim _{\substack{h \rightarrow 0 \\
n h=t-a}} h \sum_{r=0}^{n} f(t-r h)=\int_{0}^{t-a} f(t-s) d s=\int_{a}^{t} f(s) d s,
\end{aligned}
$$




$$
\begin{aligned}
{ }_{a} D_{t}^{-2} f(t) & =\lim _{\substack{h \rightarrow 0 \\
n h=t-a}} h^{2} \sum_{r=0}^{n}\left[\begin{array}{l}
2 \\
r
\end{array}\right] f(t-r h)=\lim _{\substack{h \rightarrow 0 \\
n h=t-a}} h^{2} \sum_{r=0}^{n}(r+1) f(t-r h) \\
& =\lim _{\substack{h \rightarrow 0 \\
n h=t-a}} h \sum_{r=1}^{n+1}(r h) f(t+h-r h)=\int_{0}^{t-a} s f(t-s) d s=\int_{a}^{t}(t-\tau) f(\tau) d \tau, \\
{ }_{a} D_{t}^{-3} f(t) & =\lim _{\substack{h \rightarrow 0 \\
n h=t-a}} h^{3} \sum_{r=0}^{n}\left[\begin{array}{l}
3 \\
r
\end{array}\right] f(t-r h)=\lim _{\substack{h \rightarrow 0 \\
n h=t-a}} h^{3} \sum_{r=0}^{n} \frac{(r+1)(r+2)}{2 !} f(t-r h) \\
& =\lim _{\substack{h \rightarrow 0 \\
n h=t-a}} \frac{h}{2 !} \sum_{r=1}^{n+1}(r h)^{2} f(t+h-r h)+\lim _{\substack{h \rightarrow 0 \\
n h=t-a}} \frac{h^{2}}{2 !} \sum_{r=1}^{n+1}(r h) f(t+h-r h) \\
& =\frac{1}{2 !} \int_{0}^{t-a} s^{2} f(t-s) d s=\frac{1}{\Gamma(3)} \int_{a}^{t}(t-\tau)^{2} f(\tau) d \tau
\end{aligned}
$$

Generally, for any positive integer $p$, define the $-p$ order derivative of a function $f(t)$

$$
\begin{aligned}
&{ }_{a} D_{t}^{-p} f(t)=\lim _{\substack{h \rightarrow 0 \\
n h=t-a}} h^{p} \sum_{r=0}^{n}\left[\begin{array}{l}
p \\
r
\end{array}\right] f(t-r h)=\lim _{\substack{h \rightarrow 0 \\
n h=t-a}} h^{p} \sum_{r=0}^{n} \frac{p(p+1)(p+2) \cdots(p+r-1)}{r !} f(t-r h) \\
&=\lim _{\substack{h \rightarrow 0 \\
n h=t-a}} h^{p} \sum_{r=0}^{n} \frac{p(p+1)(p+2) \cdots(p+r-1)}{(p-1) !} f(t-r h) \\
&=\lim _{\substack{h \rightarrow 0 \\
n h=t-a}} \frac{h}{(p-1) !} \sum_{r=1}^{n+1}(r h)^{p-1} f(t+h-r h) \\
&+\lim _{h \rightarrow 0} \frac{p h^{2}}{2(p-2) !} \sum_{r=1}^{n+1}(r h)^{p-2} f(t+h-r h)+\cdots+\lim _{h \rightarrow 0} h^{p} \sum_{r=t-a}^{n} f(t-r h) \\
&= \frac{1}{(p-1) !} \int_{0}^{t-a} s^{p-1} f(t-s) d s=\frac{1}{\Gamma(p)} \int_{a}^{t}(t-\tau)^{p-1} f(\tau) d \tau .
\end{aligned}
$$

Since

$$
\frac{d}{d t}\left({ }_{a} D_{t}^{-p} f(t)\right)=\frac{1}{\Gamma(p-1)} \int_{a}^{t}(t-\tau)^{p-2} f(\tau) d \tau={ }_{a} D_{t}^{-p+1} f(t)
$$

Then

$$
\begin{aligned}
{ }_{a} D_{t}^{-p} f(t) & =\int_{a}^{t}{ }_{a} D_{t}^{-p+1} f(s) d s=\int_{a}^{t} d s \int_{a}^{s}{ }_{a} D_{t}^{-p+2} f(\tau) d \tau \\
& =\int_{a}^{t} d t_{1} \int_{a}^{t_{1}} d t_{2} \int_{a}^{t_{2}}{ }_{a} D_{t}^{-p+3} f\left(t_{3}\right) d t_{3}=\int_{a}^{t} d t_{1} \int_{a}^{t_{1}} d t_{2} \cdots \int_{a}^{t_{p-1}} f\left(t_{p}\right) d t_{p} .
\end{aligned}
$$

It implies that the derivative of negative integer - $p$ order and the p-fold integral (2.21) of the continuous function $f(t)$ are equivalent.

\section{Several Fractional Derivatives}

Grunwald-Letnikov fractional derivative and integral definitions (see $[9,10])$ : Let $p$ be any arbitrary positive real number, define $p$ order derivative and $p$ order integral of a continuous function $f(t)$ :

$$
{ }_{a}^{G} D_{t}^{p} f(t)=\lim _{\substack{h \rightarrow 0 \\
n h=t-a}} h^{p} \sum_{r=0}^{n}(-1)^{r}\left(\begin{array}{l}
p \\
r
\end{array}\right) f(t-r h),
$$

and 


$$
{ }_{a}^{G} D_{t}^{-p} f(t)=\lim _{\substack{h \rightarrow 0 \\
n h=t-a}} h^{p} \sum_{r=0}^{n}\left[\begin{array}{l}
p \\
r
\end{array}\right] f(t-r h) .
$$

Riemann-Liouville fractional derivative and integral definitions (see [9]): Let $m$ be positive integer, $m<p<m+1$, define Riemann-Liouville $p$ order derivative and $p$ order integral of a continuous function $f(t)$ :

$$
{ }_{a}^{R} D_{t}^{p} f(t)=\left(\frac{d}{d t}\right)^{m+1} \int_{a}^{t}(t-\tau)^{m-p} f(\tau) d \tau,
$$

and

$$
{ }_{a}^{R} D_{t}^{-p} f(t)=\frac{1}{\Gamma(p)} \int_{a}^{t}(t-\tau)^{p-1} f(\tau) d \tau
$$

Define Caputo's fractional derivative and integral definitions(see [9]): Suppose that $f(t)$ has $m$ order continuous derivative, define Caputo's $p$ order derivative and $p$ order integral:

$$
{ }_{a}^{c} D_{t}^{p}=\frac{1}{\Gamma(m-p)} \int_{a}^{t} \frac{f^{(m)}(\tau)}{(t-\tau)^{p+1-m}} d \tau,(m-1 \leq p \leq m),
$$

and

$$
{ }_{a}^{C} D_{t}^{-p} f(t)=\frac{1}{\Gamma(p)} \int_{a}^{t}(t-\tau)^{p-1} f(\tau) d \tau .
$$

\section{Comparison of the Definition of Riemann-Liouville, Caputo and Grunwald-Letnikov}

Caputo's integral definition and Grunwald-Letnikov integral definition are consistent with the Riemann-Liouville integral definition

$$
{ }_{a}^{G} D_{t}^{-p} f(t)={ }_{a}^{R} D_{t}^{-p} f(t)={ }_{a}{ }^{C} D_{t}^{-p} f(t)=\frac{1}{\Gamma(p)} \int_{a}^{t}(t-\tau)^{p-1} f(\tau) d \tau .
$$

When $p$ is positive integer and $f^{(k)}(a)=0(k=1,2, \cdots, p)$, Caputo's derivative definition and Grunwald-Letnikov derivative definition are consistent with the Riemann-Liouville derivative definition.

$$
{ }_{a}^{G} D_{t}^{p}={ }_{a}^{R} D_{t}^{p}={ }_{a}^{C} D_{t}^{p}=f^{(p)}(t) .
$$

If $f(t)$ has $m+1$ order continuous derivative and $m<p<m+1$, then

$$
\begin{aligned}
{ }_{a}^{R} D_{t}^{p} f(t) & =\left(\frac{d}{d t}\right)^{m+1} \int_{a}^{t}(t-\tau)^{m-p} f(\tau) d \tau \\
& =\sum_{k=0}^{m} \frac{f^{(k)}(a)(t-a)^{-p+k}}{\Gamma(-p+k+1)}+\frac{1}{\Gamma(-p+m+1)} \int_{a}^{t}(t-\tau)^{m-p} f^{(m+1)}(\tau) d \tau \\
& ={ }_{a}^{G} D_{t}^{p} f(t), m<p<m+1 .
\end{aligned}
$$

It implies that Grunwald-Letnikov positive non-integer order derivative definition is consistent with the Riemann-Liouville positive non-integer order derivative definition.

\section{Summary}

Caputo's integral definition and Grunwald-Letnikov integral definition are consistent with the Riemann-Liouville integral definition. When $p$ is positive integer and $f^{(k)}(a)=0(k=1,2, \cdots, p)$, Caputo's derivative definition and Grunwald-Letnikov derivative definition are consistent with the Riemann-Liouville derivative definition. It implies that Grunwald-Letnikov positive non-integer order derivative definition is consistent with the Riemann-Liouville positive non-integer order derivative definition. 


\section{Acknowledgements}

This research was supported by National Natural Science Foundation of China (Project No. 11161018, 11561019) and Scientific Research Foundation of the Education Department of Guangxi Autonomous Region of China (No. 201106LX599).

\section{References}

[1] A. Loverro, Fractional calculus: history, definitions and applications for the engineer, Rapport technique, Univeristy of Notre Dame: Department of Aerospace and Mechanical Engineering, France, 2004.

[2] V. J. Ervin, N. Heuer and J. P. Roop, Numerical approximation of a time dependent, nonlinear, space-fractional diffusion equation, SIAM J. Numer. Anal. Vol. 45 (2007), No 2, 572-591.

[3] N. Heymans and I. Podlubny, Physical interpretation of initial conditions for fractional differential equations with Riemann-Liouville fractional derivatives, Rheologica Acta vol. 45 (2006), No. 5, 765-771.

[4] R. Gorenflo and F. Mainardi, Random walk models for space-fractional diffusion processes, Fractional Calulus and Applied Analysis, vol. 1 (1998), No 2, 167-191.

[5] E. Gerolymatou, I. Vardoulakis and R. Hilfer, Modelling infiltration by means of a nonlinear fractional diffusion model, J. Phys. D: Appl. Phys. Vol. 39 (2006) 4104-4110.

[6] K. Oldham, J. Spanier, The Fractional Calculus, Theory and Applications of Differentiation and Integration of Arbitrary Order, Academic Press, USA, 1974.

[7] G. Samko, A. A. Kilbas, Marichev, Fractional Integrals and Derivatives: Theory and Applications, Gordon and Breach, Switzerland, 1993.

[8] K.S. Miller, An Introduction to Fractional Calculus and Fractional Differential Equations, J. Wiley and Sons, New York, 1993.

[9] I. Podlubny, Fractional Differential Equations, Academic Press, New York, 1999.

[10]A. V. Letnikov, Theory of differentiation of arbitrary order, Mat. Sb. vol. 3 (1868) 1-68 (in Russian).

[11] R. Khalil, M. Al Horani, A. Yousef and M. Sababheh, A new Denition of Fractional Derivative, J. Comput. Appl. Math. Vol. 264(2014) 65-70.

[12] T. Abdeljawad, On conformable fractional calculus, J. Computational Appl. Math. Vol. 279(2015) 57-66. 